\title{
Portfolio Selection with Heavy Tails
}

\author{
Namwon Hyung and Casper G. de Vries* \\ University of Seoul, Tinbergen Institute, \\ Erasmus Universiteit Rotterdam ${ }^{\dagger}$ and EURANDOM
}

July 2004

\begin{abstract}
Consider the portfolio problem of choosing the mix between stocks and bonds under a downside risk constraint. Typically stock returns exhibit fatter tails than bonds corresponding to their greater downside risk. Downside risk criteria like the safety first criterion therefore often select corner solutions in the sense of a bonds only portfolio. This is due to a focus on the asymptotically dominating first order Pareto term of the portfolio return distribution. We show that if second order terms are taken into account, a balanced solution emerges. The theory is applied to empirical examples from the literature.
\end{abstract}

Key words: safety first, heavy tails, portfolio diversification; JEL code: G11

*Both authors like to thank the Tinbergen Institute for support. Hyung is gratefully acknowledges support by the Korea Research Foundation Grant (KRF-2003-003-B00103).

${ }^{\dagger}$ Corresponding author: Casper G. de Vries, Department of Accounting and Finance H1425, Erasmus Univeristy Rotterdam, PO Box 1738, 3000 DR, Rotterdam, The Netherlands, email <cdevries@few.eur.nl>. 


\section{Introduction}

Consider the portfolio problem of choosing the mix between a stock index and a government bond index. The mean variance criterion selects non-zero proportions of each as long as stocks have higher expected returns and higher variance. Investors nevertheless in addition often worry about the downside risk features of their portfolio, witness the popularity of policies with put protection that lock in gains, portfolio insurance, capital buffers at pension funds, Value at Risk (VaR) exercises at banks, etc. It is a fact that asset return distributions exhibit fat tails, i.e. are asymptotic to a Pareto distribution. Typically stocks exhibit fatter tails than bonds, i.e. have smaller hyperbolic Pareto coefficient, corresponding to the greater downside risk of stocks. Downside risk criteria like the safety first criterion therefore often select corner solutions in the sense of a bonds only portfolio. This is due to a focus on the tail of the asset return distributions whereby only the asymptotically dominating first order Pareto term is taken into account. In this note we show that if the second order terms are considered as well, a more balanced solution emerges. The theory is applied to examples from the literature.

Portfolio risk and its upside potential are in an important way driven by the 'abnormal' returns emanating from heavy-tailed distributed asset returns. Therefore the financial industry often employs so called downside risk measures to characterize the asset and portfolio risk, since it is widely recognized that large losses are more frequent than a normal distribution based statistic like the standard deviation suggests. A formal portfolio selection criterion which incor- 
porates the concern for downside risk is the safety first criterion, see Roy (1952) and Arzac and Bawa (1977). The paper by Gourieroux, Laurent and Scaillet (2000) analyzes the sensitivity of VaR with respect to portfolio allocation, which is essentially the same problem as portfolio selection with the safety first criterion. Gourieroux et al. (2000) show how to check for the convexity of the estimated VaR efficient portfolio set. Jansen, Koedijk and de Vries (2000) apply the safety first criterion and exploit the fact that returns are fat-tailed. They propose a semi-parametric method for modeling tail events and use extreme value theory to measure the downside risk. This method was subsequently used by Susmel (2001) in an application involving Latin American stock markets.

If one selects assets on the basis of the tail properties of the return distribution, there is a tendency to end up with a corner solution whereby the asset with the highest tail coefficient (thinnest tail) is selected, see e.g. Straetmans (1998, ch.5), Jansen et al. (2000), Hartmann, Straetmans and de Vries (2000) and Poon, Rockinger and Tawn (2003). This follows from Geluk and de Haan (1987), who show that a convolution of two regularly varying variables produces a random variable which has the same tail properties as the fattest tail of the two convoluting variables, i.e. the fattest tail (lowest tail coefficient) dominates. In case the tails are equally fat, the scales of the two random variables has to be added. In this paper we show how to extend the first order convolution result to a second order asymptotic expansion. Whereas in the first order convolution result only the fattest of the two tails plays a role, in the second order expansion often both tails play a role. We show that with a second order expansion 
of the downside risk, the portfolio solution yields a balanced solution, i.e. both assets are held in non-zero proportion, whereas the first order expansion selects the corner solution. In the empirical application, we follow up on Jansen et al. (2000) and Susmel (2001), who apply the safety first criterion to a number of portfolio problems. In several cases Jansen et al. (2000) end up with a corner solution. We calculate the downside risk using the second order expansion and show how this implies a move towards the interior.

\section{Extreme Value Theory}

The fat tail property is one of the salient features of asset returns. This can be modeled by letting the tail of the distribution be governed by a power law, instead of an exponential rate. Technically speaking, suppose that the returns are i.i.d. and have tails which vary regularly at infinity. This entails that to a first order

$$
P\{X>s\}=A s^{-\alpha}+o\left(s^{-\alpha}\right)
$$

as $s \rightarrow \infty$, where $\alpha>0, A>0$. A more detailed parametric form for the tail probability can be obtained by taking a second order expansion at infinity. There are only two non-trivial expansions (de Haan and Stadtmüller, 1996). The first expansion has a second order term which also declines hyperbolically

$$
P\{X>s\}=A s^{-\alpha}\left[1+B s^{-\beta}+o\left(s^{-\beta}\right)\right]
$$


as $s \rightarrow \infty$, where $\alpha>0, A>0, \beta>0$ and $B$ is a real number. This expansion applies to the non-normal sum-stable, Student-t, Fréchet, and other fat tailed distributions. The other non-trivial expansion is

$$
P\{X>s\}=A s^{-\alpha}[1+B \log s+o(\log s)]
$$

which is not considered in this paper ${ }^{1}$.

We assume that the tails of two assets are different but symmetric, and vary regularly at infinity. Consider the following second order expansion,

$$
\begin{aligned}
& P\left\{X_{1}>s\right\}=P\left\{X_{1}<-s\right\}=A_{1} s^{-\alpha_{1}}\left[1+B_{1} s^{-\beta_{1}}+o\left(s^{-\beta_{1}}\right)\right] \\
& P\left\{X_{2}>s\right\}=P\left\{X_{2}<-s\right\}=A_{2} s^{-\alpha_{2}}\left[1+B_{2} s^{-\beta_{2}}+o\left(s^{-\beta_{2}}\right)\right]
\end{aligned}
$$

as $s \rightarrow \infty$. We assume $2<\alpha_{1} \leq \alpha_{2}$. The assumption of $2<\alpha_{1}$ implies that at least the mean and variance exist, which seems to be the relevant case for financial data. Portfolios are essentially (weighted) sums of different random variables. We therefore investigate the tail probability of the convolution $X_{1}+$ $X_{2}$. The case of equal tail indices $\alpha_{1}=\alpha_{2}$ is known from Feller (1971, ch. VIII). In this case $P\left\{X_{1}+X_{2}>s\right\}=\left(A_{1}+A_{2}\right) s^{-\alpha_{1}}+o\left(s^{-\alpha_{1}}\right)$ as $s \rightarrow \infty$. When the tail indices are unequal we have the following results.

Theorem 1 Suppose that the tails of the distributions of $X_{1}$ and $X_{2}$ satisfy (1) and (2). Moreover, assume $2<\alpha_{1}<\alpha_{2}$ so that $E[X]$ and $E\left[X^{2}\right]$ are

\footnotetext{
${ }^{1}$ The slow decay of the second order term makes this class sufficiently different from the other class. The inclusion of this class would make our paper overly long.
} 
bounded. When $X_{1}$ and $X_{2}$ are independent, the asymptotic 2-convolution up to the second order terms is

(I) if $\alpha_{2}-\alpha_{1}<\min \left(\beta_{1}, 1\right)$,

then $P\left\{X_{1}+X_{2}>s\right\}=A_{1} s^{-\alpha_{1}}+A_{2} s^{-\alpha_{2}}+o\left(s^{-\alpha_{2}}\right)$

(II) if $1<\alpha_{2}-\alpha_{1}$ and $1<\beta_{1}$,

then $P\left\{X_{1}+X_{2}>s\right\}=A_{1} s^{-\alpha_{1}}+A_{1} \alpha_{1} E\left[X_{2}\right] s^{-\alpha_{1}-1}+o\left(s^{-\alpha_{2}}\right)$

(III) if $\beta_{1}<\alpha_{2}-\alpha_{1}$ and $\beta_{1}<1$,

then $P\left\{X_{1}+X_{2}>s\right\}=A_{1} s^{-\alpha_{1}}+A_{1} B_{1} s^{-\alpha_{1}-\beta_{1}}+o\left(s^{-\alpha_{1}-\beta_{1}}\right)$

(IV) if $\alpha_{2}-\alpha_{1}=1<\beta_{1}$,

then $P\left\{X_{1}+X_{2}>s\right\}=A_{1} s^{-\alpha_{1}}+\left\{A_{2}+A_{1} \alpha_{1} E\left[X_{2}\right]\right\} s^{-\alpha_{2}}+o\left(s^{-\alpha_{2}}\right)$

(V) if $\alpha_{2}-\alpha_{1}=\beta_{1}<1$,

then $P\left\{X_{1}+X_{2}>s\right\}=A_{1} s^{-\alpha_{1}}+\left\{A_{2}+A_{1} B_{1}\right\} s^{-\alpha_{2}}+o\left(s^{-\alpha_{2}}\right)$

(VI) if $\alpha_{2}-\alpha_{1}=\beta_{1}=1$,

then $P\left\{X_{1}+X_{2}>s\right\}=A_{1} s^{-\alpha_{1}}+\left\{A_{2}+A_{1} \alpha_{1} E\left[X_{2}\right]+A_{1} B_{1}\right\} s^{-\alpha_{2}}+$ $o\left(s^{-\alpha_{2}}\right)$.

Proof. We only provide the proof of the upper tail case. The proof for the lower tail case only requires a small modification of this proof. Parts of the proof are similar in spirit as the proof in Dacarogna, Müller, Pictet and de Vries (2001, Lemma 4). It is an extension of Feller's original convolution result for regularly varying distributions. We divide the area over which we have to integrate into five parts $A, B, C, D$ and $E$; where $P\{A\}=P\left\{X_{1}+X_{2} \leq s\right.$, $\left.X_{1}>-\frac{s}{2}, X_{2}>\frac{s}{2}\right\}, P\{C\}=P\left\{X_{1} \leq \frac{s}{2}, X_{2} \leq \frac{s}{2}\right\}, P\{D\}=P\left\{X_{1}+X_{2} \leq\right.$ $\left.s, X_{1} \leq-\frac{s}{2}, X_{2}>\frac{s}{2}\right\}$, and where $P\{B\}$ and $P\{E\}$ are the counterparts of 
$P\{A\}$ and $P\{D\}$ respectively. By integration we find $P\{A\}, P\{D\}$, and $P\{C\}$.

The integrals are provided in Appendix A. Adding up and ignoring the terms which are of smaller order, like $s^{-2 \alpha_{1}}$, we find that

$$
\begin{aligned}
P\left\{X_{1}+X_{2}>s\right\} & \approx 1-[P\{C\}+P\{A\}+P\{B\}] \\
& \approx A_{1} s^{-\alpha_{1}}+A_{1} B_{1} s^{-\alpha_{1}-\beta_{1}}+A_{2} s^{-\alpha_{2}}+A_{2} B_{2} s^{-\alpha_{2}-\beta_{2}} \\
& +A_{1} \alpha_{1} E\left[X_{2}\right] s^{-\alpha_{1}-1}+A_{1} B_{1}\left(\alpha_{1}+\beta_{1}\right) E\left[X_{2}\right] s^{-\alpha_{1}-\beta_{1}-1} \\
& +A_{2} \alpha_{2} E\left[X_{1}\right] s^{-\alpha_{2}-1}+A_{2} B_{2}\left(\alpha_{2}+\beta_{2}\right) E\left[X_{1}\right] s^{-\alpha_{2}-\beta_{2}-1} \\
& +A_{1} \frac{\left(\alpha_{1}+1\right) \alpha_{1}}{2} E\left[X_{2}^{2}\right] s^{-\alpha_{1}-2} \\
& +A_{2} \frac{\left(\alpha_{2}+1\right) \alpha_{2}}{2} E\left[X_{1}^{2}\right] s^{-\alpha_{2}-2} .
\end{aligned}
$$

By considering the different parameter configurations (I) - (VI), we obtain the results of Theorem 1.

What is the relevance of this theorem for portfolio selection? Suppose that portfolio selection is done on the basis of the concern for the downside risk, safety-first criterion using this convolution result. By mapping negative returns into the positive quadrant, this theorem applies to the left tail with a little modification. Let $X_{i}$ denote the loss returns on two independent project. Under this criterion the problem is to minimize $P\left\{\omega X_{1}+(1-\omega) X_{2}>s\right\}$ at some large loss levels $s$ by choosing the asset mix $\omega$. Suppose only the first order terms of tail probability $P\left\{X_{i}>s\right\}=A_{i} s^{-\alpha_{i}}$ are taken into account. Then for large loss levels $s$ one choose $\omega=0$, if $\alpha_{1}<\alpha_{2}$. This corner solution is driven by evaluation of the safety first criterion in the limit (where only the first order 
term is relevant). In practice what counts are very high, but finite loss levels. Thus a second order expansion in which the second order term still plays a role has practical relevance. To this end we can use the Theorem 1.

Consider first the case III above. Since asset 1 dominates the first two terms in the loss probability, one is still better of by putting all eggs in one basket. Turn to case I. If one would focus on the first term only, i.e. only taking the limit as $s \rightarrow \infty$ into consideration, then again only asset two is selected. At any finite loss level $s$, this solution is, however, suboptimal. Given that $P\left\{X_{1}+X_{2}>s\right\} \approx A_{1} s^{-\alpha_{1}}+A_{2} s^{-\alpha_{2}}$ in case I, one should take both assets into account and diversify away from the corner solution. This lowers the loss probability $P\left\{X_{1}+X_{2}>s\right\}$ at any finite loss level $s$. This idea is put on a firm footing in the next section by investigating the convexity properties of the solutions.

\section{The Sensitivity and Convexity of VaR}

The aim of this section is to analyze the sensitivity of VaR with respect to portfolio allocation. Gourieroux et al. (2000) derive analytical expression for the first and second derivatives of the VaR in a general framework, and state sufficient conditions for the VaR efficient portfolio set to be convex. Gourieroux et al. (2000) also provide explicit expression for the first and second derivatives in case of the normal distribution. Here we provide explicit expressions for the class of fat tailed distributions. Moreover, we show how to ensure an interior 
solution under which the VaR is convex with respect to the portfolio weight. If a risk measure is a convex function of the portfolio allocation, it induces portfolio diversification. From this we can ensure that an interior solution to the safety first problem exists. While Gourieroux et al. (2000) show the convexity of the VaR-efficient portfolio set in general, they do not give conditions to ensure an interior solution for the optimal allocation.

First, we derive analytical expression of derivatives of the tail probability at a given quantile in the heavy tail context. This allows us to discuss the convexity properties of VaR. We consider two financial assets whose returns at time $t$ are denoted by $X_{i}, i=1,2$. We suppress time indices whenever this is not confusing. The return at $t$ of a portfolio with allocation $\omega$ is then $\omega X_{1}+(1-\omega) X_{2}$. For a loss probability level $p$ the Value at Risk, $\operatorname{VaR}(\omega, p)$ is defined by:

$$
P\left\{\omega X_{1}+(1-\omega) X_{2}>\operatorname{VaR}(\omega, p)\right\}=p .
$$

In practice, VaR is often computed under the normality assumption for returns. Recently, semi-parametric approaches have been developed, which are based on the extreme value approximation to the tail probability like in the previous section. We compute the first and second derivatives of the probability with respect to portfolio allocation under this approximation. Under the safety first rule an investor specifies a low threshold return $s$ and selects the portfolio of assets which minimizes the probability of a return below this threshold. 


\subsection{Convexity of the Tail Probability}

Suppose the tails of the distributions of $X_{1}$ and $X_{2}$ satisfy (1) and (2). We obtain the first and second derivatives in the proof to Lemma 1. We first investigate the case I from the convolution Theorem 1.

Lemma 1 Under assumptions of Theorem 1 and if $\alpha_{2}-\alpha_{1}<\min \left(\beta_{1}, 1\right)$, there exists a $\omega^{*} \in(0,1)$ for given large $s>0$ such that

$$
P\left\{\omega^{*} X_{1}+\left(1-\omega^{*}\right) X_{2}>s\right\} \leq P\left\{\omega X_{1}+(1-\omega) X_{2}>s\right\}
$$

for any $0 \leq \omega \leq 1$. The equality holds only when $\omega=\omega^{*}$.

Proof. From Theorem 1, the asymptotic 2-convolution up to the second order terms is

$$
\begin{aligned}
P\left\{\omega X_{1}+(1-\omega) X_{2}\right. & >s\} \approx \omega^{\alpha_{1}} A_{1} s^{-\alpha_{1}}+(1-\omega)^{\alpha_{2}} A_{2} s^{-\alpha_{2}} \\
& =p(\omega, s),
\end{aligned}
$$

for given large $s>0$. We show the function of $p(\omega, s)$ has a minimum for some $\omega \in(0,1)$. The slope of this function with respect to $\omega$ is

$$
\frac{\partial p(\omega, s)}{\partial \omega}=\alpha_{1} \omega^{\alpha_{1}-1} A_{1} s^{-\alpha_{1}}-\alpha_{2}(1-\omega)^{\alpha_{2}-1} A_{2} s^{-\alpha_{2}}
$$


for large $s>0$. Thus slopes at the endpoints are

$$
\left.\frac{\partial p(\omega, s)}{\partial \omega}\right|_{\omega=0}=-\alpha_{2} A_{2} s^{-\alpha_{2}}<0
$$

and

$$
\left.\frac{\partial p(\omega, s)}{\partial \omega}\right|_{\omega=1}=\alpha_{1} A_{1} s^{-\alpha_{1}}>0
$$

for large $s>0$. The slope of this function increases monotonically since the second order derivative of this function is

$$
\frac{\partial^{2} p(\omega, s)}{\partial \omega^{2}}=\left(\alpha_{1}-1\right) \alpha_{1} \omega^{\alpha_{1}-2} A_{1} s^{-\alpha_{1}}+\left(\alpha_{2}-1\right) \alpha_{2}(1-\omega)^{\alpha_{2}-2} A_{2} s^{-\alpha_{2}}
$$

which is positive for all $0 \leq \omega \leq 1$ provided $\alpha=\min \left\{\alpha_{1}, \alpha_{2}\right\}>1$.

In the proof of the Lemma 1 we show the convexity of $\omega^{\alpha_{1}} A_{1} s^{-\alpha_{1}}+(1-\omega)^{\alpha_{2}} A_{2} s^{-\alpha_{2}}$. Note that this expression is only asymptotic to $P\left\{\omega X_{1}+(1-\omega) X_{2}>s\right\}$ as $s \rightarrow \infty$. Therefore $\partial P\left\{\omega^{*} X_{1}+\left(1-\omega^{*}\right) X_{2}>s\right\} / \partial \omega$ will typically be close to zero but not be exactly equal to zero.

Remark 1 The Lemma 1 implies that if one constructs a portfolio which minimizes the probability of extreme negative returns, one has to assign some weight to the asset with the fatter tail.

Remark 2 Under conditions (II) and (III) from Theorem 1, Lemma 1 has trivial solutions such as $\omega^{*}=0$ or $\omega^{*}=1$ depending on the conditions of parameters.

Remark 3 With conditions (IV), (V) and (VI) from Theorem 1, Lemma 1 
has non-trivial solution such that $\omega^{*} \in(0,1)$ provided the parameters satisfy additional conditions. We illustrate the case of condition (IV) as an example. Under the condition (IV), $\alpha_{2}-\alpha_{1}=1<\beta_{1}$, then $P\left\{\omega X_{1}+(1-\omega) X_{2}>s\right\} \approx$ $\omega^{\alpha_{1}} A_{1} s^{-\alpha_{1}}+(1-\omega)^{\alpha_{2}} A_{2} s^{-\alpha_{2}}+\omega^{\alpha_{1}} A_{1} \alpha_{1} E\left[(1-\omega) X_{2}\right] s^{-\alpha_{2}} \equiv q(\omega)$. The slope of this function is

$$
\begin{aligned}
\frac{\partial q(\omega)}{\partial \omega} & =\omega^{\alpha_{1}-1} \alpha_{1} A_{1} s^{-\alpha_{1}}-(1-\omega)^{\alpha_{2}-1} \alpha_{2} A_{2} s^{-\alpha_{2}} \\
& +\left(\omega^{\alpha_{1}-1} \alpha_{1}-\omega^{\alpha_{1}}\left(\alpha_{1}+1\right)\right) A_{1} \alpha_{1} E\left[X_{2}\right] s^{-\alpha_{2}}
\end{aligned}
$$

For the corner solution excluding the asset 1 with the heaviest tail

$$
\left.\frac{\partial q(\omega)}{\partial \omega}\right|_{\omega=0}=-\alpha_{2} A_{2} s^{-\alpha_{2}}<0
$$

for large $s>0$. On the other hand, if the following condition is satisfied for large $s>0$,

$$
\left.\frac{\partial q(\omega)}{\partial \omega}\right|_{\omega=1}=\alpha_{1} A_{1} s^{-\alpha_{1}}-\alpha_{1} A_{1} E\left[X_{2}\right] s^{-\alpha_{2}}>0
$$

then there exists a non-trivial solution under the condition (IV), too. The last condition will be satisfied if $E\left[X_{2}\right]<s$. That is, $E\left[X_{2}\right]$ must not be too large for the given a finite loss level s. This holds certainly as long as the expected return is positive (since the $E\left[X_{2}\right]<0$, recall that a positive $X_{i}$ reflects a loss). 


\subsection{Convexity of VaR}

We now turn around the question from the previous section, and ask whether the VaR at a given probability level is convex. If the VaR criterion is used as the risk measure for judging the portfolio, and if we can show that the VaR is a convex function of the portfolio allocation, then there is an incentive for portfolio diversification under the VaR objective.

Lemma 2 Under assumptions of Theorem 1 and if $\alpha_{2}-\alpha_{1}<\min \left(\beta_{1}, 1\right)$, consider the downside risk level

$P\left\{\omega X_{1}+(1-\omega) X_{2}>s\right\}=\omega^{\alpha_{1}} A_{1} s^{-\alpha_{1}}\left[1+\frac{(1-\omega)^{\alpha_{2}} A_{2}}{\omega^{\alpha_{1}} A_{1}} s^{-\alpha_{2}+\alpha_{1}}+o\left(s^{-\alpha_{2}+\alpha_{1}}\right)\right]$

and define the VaR implicitly as follows $P\left\{\omega X_{1}+(1-\omega) X_{2}>\operatorname{VaR}(\omega, p)\right\}=$ p. By De Bruijn's theory on asymptotic inversion

$$
\operatorname{VaR}(\omega, p)=\omega A_{1}^{\frac{1}{\alpha_{1}}} p^{-\frac{1}{\alpha_{1}}}\left[1+\frac{(1-\omega)^{\alpha_{2}}}{\omega^{\alpha_{2}}} \frac{A_{2}}{\alpha_{1} A_{1}^{\alpha_{2} / \alpha_{1}}} p^{\frac{\alpha_{2}-\alpha_{1}}{\alpha_{1}}}+o(1)\right]
$$

for any $0<\omega<1$.

Proof. Directly follows from de Bruijn's inverse in Theorem 1.5.13 of Bingham, Goldie and Teugels (1987).

For the given loss probability $p$, we can find an allocation which minimizes the VaR risk.

Lemma 3 Under assumptions of Theorem 1 and if $\alpha_{2}-\alpha_{1}<\min \left(\beta_{1}, 1\right)$, there 
exist $\omega^{*} \in(0,1)$ for given probability level $\bar{p}$ such that

$$
\operatorname{VaR}\left(\omega^{*}, \bar{p}\right) \leq \operatorname{VaR}(\omega, \bar{p})
$$

for any $0<\omega<1$. The equality holds only when $\omega=\omega^{*}$.

Proof. For a given probability level $\bar{p}$, the first derivative of the VaR is

$$
\begin{aligned}
\frac{\partial V a R(\omega, \bar{p})}{\partial \omega} & =A_{1}^{\frac{1}{\alpha_{1}}} \bar{p}^{-\frac{1}{\alpha_{1}}}-\alpha_{1}^{-1} A_{1}^{\frac{1-\alpha_{2}}{\alpha_{1}}} A_{2} \bar{p}^{\frac{\alpha_{2}-\alpha_{1}-1}{\alpha_{1}}}\left\{\alpha_{2}(1-\omega)^{\alpha_{2}-1} \omega^{1-\alpha_{2}}\right. \\
& \left.+\left(\alpha_{2}-1\right)(1-\omega)^{\alpha_{2}} \omega^{-\alpha_{2}}\right\} .
\end{aligned}
$$

From this, it follows that

$$
\left.\frac{\partial \operatorname{VaR}(\omega, \bar{p})}{\partial \omega}\right|_{\omega=1}=A_{1}^{\frac{1}{\alpha_{1}}} \bar{p}^{-\frac{1}{\alpha_{1}}}>0 .
$$

Moreover, multiplying the derivative by $\omega^{\alpha_{2}}$ and evaluating the resulting expression at $\omega=0$ gives

$$
\left.\omega^{\alpha_{2}} \frac{\partial V a R(\omega, \bar{p})}{\partial \omega}\right|_{\omega=0}=-\alpha_{1}^{-1} A_{1}^{\frac{1-\alpha_{2}}{\alpha_{1}}} A_{2} \bar{p}^{\frac{\alpha_{2}-\alpha_{1}-1}{\alpha_{1}}}\left(\alpha_{2}-1\right)<0 .
$$

The second-order derivative at $\omega=\omega^{*}$ with respect to the portfolio allocation is:

$$
\frac{\partial^{2} V a R(\omega, \bar{p})}{\partial \omega^{2}}=\frac{\alpha_{2}\left(\alpha_{2}-1\right)}{\alpha_{1}} A_{1}^{\frac{1-\alpha_{2}}{\alpha_{1}}} A_{2} \bar{p} \frac{\alpha_{2}-\alpha_{1}-1}{\alpha_{1}} \omega^{-3}\left(\frac{1}{\omega}-1\right)^{\alpha_{2}-2}
$$

which is strictly positive for $\omega \in(0,1)$ under the stated assumptions. Together these derivatives imply there is an interior minimum. 
It follows that the VaR is convex in the portfolio mix if the distribution of returns have tails which vary regularly at infinity. The VaR criterion thus induces diversification, even though it penalizes asset returns which have a higher asymptotic downside risk than others. Under the stated conditions in Lemma 3, the optimal choice includes the riskier asset for the limited downside risk portfolio.

\section{Revisit to Jansen et al. (2000)}

We now demonstrate the relevance of the above second order expansion by revisiting applications from the literatures. It will be shown how the second order theory modifies the portfolio selected if one only relies on the first order theory. An example is a study of the safety first criterion by Jansen et al. (2000). We first briefly review the safety first criterion and then present our portfolio choices.

\subsection{Safety-first portfolio}

Portfolio selection is based on a trade-off between expected return and risk. The risk in the safety-first criterion, initially proposed by Roy (1952) and Arzac and Bawa (1977), is evaluated by the probability of failure. A lexicographic form of the safety first principle is:

$$
\max _{\omega_{i}, b}(\pi, \mu) \text { lexicographically, }
$$


subject to

$$
\sum_{i} \omega_{i} V_{i t}+b=W_{t}
$$

where $\pi=1$ if $p=P\left\{\sum \omega_{i} V_{i t+1}+b r \leq s\right\} \leq \delta$, and $\pi=1-p$ otherwise. Furthermore let $\mu=E\left[\sum \omega_{i} V_{i t+1}\right]+b r, V_{i t}$ denotes the initial market values of asset $i$ at time $t, W_{t}$ is the initial wealth level of the investor, $b$ denotes the amount of lending or borrowing $(b>0$ represents lending), $r$ is the risk-free gross rate of return, $\omega_{i}$ denotes the weight of invested amount in the risky asset $i, s$ is the disaster level of wealth, and $\delta$ gives the maximal acceptable probability of this disaster.

Arzac and Bawa (1977) showed that the safety first problem can be separated into two problems: First, the risk averse safety-first investor maximizes the ratio of the risk premium to the return opportunity loss that he is willing to incur with probability $\delta$, that is

$$
\max _{\omega_{i}} \frac{(\bar{R}-r)}{\left(r-q_{\delta}(R)\right)}
$$

where $R=\sum \omega_{i} V_{i t+1} / \sum \omega_{i} V_{i t}$ are the gross returns, $\bar{R}=E(R)$, and $q_{\delta}(R)$ is a quantile (loss level) such that there is $\delta \%$ probability of returns less than or equal to this value, that is, the VaR. In the second stage the investor determines the scale of the risky portfolio and the amount borrowed from the budget constraint;

$$
W_{t}-b=\frac{s-r W_{t}}{q_{\delta}(R)-r}
$$

For further details on this part, we refer to Arzac and Bawa (1977). 


\subsection{Empirical illustrations}

We re-calculate the optimum portfolio weights for the examples in Jansen et al. (2000) which resulted in a corner solution. By using Lemma 1 and the parameter estimates from Jansen et al. (2000) we obtain an interior solution when we apply the second order theory. The problem consists in choosing between investing in a mutual fund of bonds or a mutual fund of stocks over the period 1926.01 - 1992.12 with 804 monthly observations of a US bond index and a US stock index (from the CRSP database). We also present, separately, an analysis of the two French stocks Thomson-CSF and L'Oreal, covering 546 daily observations, studied both by Jansen et al. (2000) and Gourieroux et al. (2000).

The Table 1 reproduces the summary statistic and tail indices from Jansen et al. (2000). For US assets the tail index is calculated for the lower tails of the distribution of monthly stock and bond returns. For the daily returns of the two French stocks the calculations combined the data from the upper and lower tails upon the assumption of tail symmetry.

From Table 1 we see that the first order tail indices differ. In Jansen et al. (2000) for the case of the two French stocks the safety first criterion allocates all wealth to L'Oreal which has the higher tail index. For the US assets, note that with $r=1$ and and a risk level $\delta=0.000625$ all wealth is allocated to the low risk (higher tail index) bond. Our solutions using the second order approach will be different.

We verify whether the conditions for an interior solution from Lemma 1 do apply. Without loss of generality, we set US stock and Thomson-CSF as $X_{1}$. 
We calculate the second order tail index, $\beta_{1}$, by using the estimates from Table 1. One can calibrate the values of the second order coefficient from Table 1 as follows. A consistent estimator for the ratio between the first and second order tail indices is

$$
\widehat{\beta / \alpha}=\frac{\ln \hat{m}}{2 \ln n-2 \ln \hat{m}},
$$

where $n$ is the number of observations, $m$ is the window size for the estimation of the tail index, see Danielsson et al. (2000). By Proposition 1.7 from Geluk and de Haan (1987) on the properties of regularly varying functions we have that $\frac{\ln \hat{m}}{\ln n} \rightarrow \frac{2 \beta / \alpha}{1+2 \beta / \alpha}$ in probability as $n \rightarrow \infty$. Then we use the fact that $\hat{m} / m \rightarrow 1$ in probability, where $\hat{m}$ is a consistent estimator of $m$. Thus, for the US assets, $\beta_{1}=0.809$ and $\alpha_{2}-\alpha_{1}=0.311$, in case of the two French stocks, $\beta_{1}=1.657$ and $\alpha_{2}-\alpha_{1}=0.459$. Thus both cases satisfy the conditions of Lemma 1 .

To determine the portfolio mix, we follow the same procedure as in Jansen et al. (2000). We first calculate the VaR quantiles for each hypothetical portfolio ${ }^{2}$. These are reported in Table 2. The investor can borrow or lend at the risk-free rate $r$, and maximizes $(\bar{R}-r) /\left(r-q_{\delta}(R)\right)$. The safety-first investor specifies the desired probability $\delta$ level; the calculations are done for two choices of $\delta, \delta=0.0025$, and $\delta=0.000625$. Two interest rates are used, $r=1$ and

\footnotetext{
${ }^{2}$ We can calculate $A_{i}, i=1,2$, used in Jansen et al. (2000) by using

$$
A_{i}=\frac{m_{i}}{n} X_{\left(m_{i}\right)}^{\alpha_{i}}
$$

where $X_{(m)}$ is the $m$-th largest observation. Then we plug those values in Lemma 1 , and solve the following approximation

$$
\omega^{\alpha_{1}} A_{1} q_{\delta}^{-\alpha_{1}}+(1-\omega)^{\alpha_{2}} A_{2} q_{\delta}^{-\alpha_{2}} \approx \delta
$$

to get the value $q_{\delta}$ for the given value of $\omega$ and $\delta$.
} 
$r=1.00303$ (the latter corresponds to an annual rate of $3.7 \%$, which equals the average returns on the US Treasury bills over 1926-1992). The mean return $\bar{R}$ is taken from Table 2 by weighting the mean returns on the two assets with the indicated portfolio mix. Optimal portfolios in Table 3 are marked with an asterisk. In all four configurations considered, the optimal portfolio contains $20 \%$ stocks and $80 \%$ bonds. Figure 1 illustrates the portfolio choice problem, plotting the mean return versus VaR for portfolios of stocks and bonds when $r=1.003$. For the case $r=1$ and $\delta=0.000625$, Jansen et al.(2000) select a corner solution with $100 \%$ bonds. In our procedure, however, stocks are still part of the portfolio.

Empirical analyses of the daily data on the two French stocks are presented in Tables 2 and Table 4. Figure 2 illustrates that the limited downside risk portfolio selection criterion chooses a portfolio with $30 \%$ of Thomson-CSF stocks and $70 \%$ of L'Oreal stocks, not the corner solution as in Jansen et al. (2000).

To conclude, if we take into account the second order terms, solutions are often bounded away from the $100 \%$ bond portfolio in the example of US assets, while if only the first order terms are taken into account, a corner solution is repeatedly selected. This may make the portfolio overly conservative, giving up quite a bit of upside potential.

We briefly examine another example from the literature. Susmel (2001) investigates the diversification opportunities which the Latin American emerging markets offer to a US safety first investor. From the portfolio choice problem between an equally weighted Latin American Index and US index, the optimal 
investment in the Latin American Index is $15 \%$ in Susmel's (2001) paper. Instead of an equally weighted Latin American Index, we analyze the optimum portfolio weight for each pair of US and Argentina, US and Brazil, US and Chile, US and Mexico respectively. One can verify that the conditions of Lemma 1 are satisfied for all Latin American stocks combined with US from the estimates in Table 4 of Susmel (2001). Using the same procedure as before, we calculate optimal weights for each pair. For the case of $r=1$ and $\delta=0.00289(1 / 346)$, we find only portfolio weights $1 \%, 2 \%, 5 \%$ and $2 \%$. For the case of $r=1$ and $\delta=0.001445(0.5 / 346)$, we find only $1 \%, 1 \%, 4 \%$ and $2 \%$ portfolio weightings. These low proportions of Latin American stocks are due to the much higher tail risk (low tail indices) compared to the US. ${ }^{3}$ Since the estimated tail indices of US and Latin American markets are very different, from 3.2 to $1.8 \sim 2.1$ the portfolio selection problems have near corner solutions for all cases.

\section{Conclusion}

We consider the portfolio problem of choosing the mix between stocks and bonds. Investors often worry about the downside risk features of their portfolio. It is a fact that asset return distributions exhibit fat tails, i.e. are asymptotic to a Pareto distribution. Typically stocks exhibit fatter tails than bonds corresponding to the greater downside risk of stocks. Downside risk criteria like

\footnotetext{
${ }^{3}$ Susmel (2001) proceeds along a different line and selects much higher proportions. The reason is that Susmel (2001) estimates different tail indices for each portfolio combination. This approach, however, biases the tail indices upward (causing understimation of the risk). This further clarified in the Appendix B.
} 
the safety first criterion therefore often select corner solutions in the sense of a bonds only portfolio. This is due to a focus on the tail of the asset return distributions whereby only the asymptotically dominating first order Pareto term is taken into account.We extend the first order convolution result to a second order asymptotic expansion. Whereas in the first order convolution result only the fattest of the two tails plays a role, in the second order expansion often the tails of both assets play a role. We suggest that with a second order expansion of the downside risk, the portfolio solution may yield a balanced solution, i.e. both assets are held in non-zero proportion, whereas the first order expansion selects the corner solution.

In the empirical application, we follow up on Jansen et al. (2000), who apply the safety first criterion to a number of portfolio problems. In the cases where Jansen et al. (2000) give a corner solution, our procedure still selects both assets for incorporation in the limited downside risk portfolio. We also briefly addressed another example from the literature.

In this paper and the related literatures, the independence between assets was assumed, which is not completely realistic for financial assets. This assumption can be weakened. For instance, we can allow cross-sectional dependency by using Capital Asset Pricing Model (CAPM) from finance. Then we can divide the risk of assets into the market risk component and the idiosyncratic risk. Such an extension is under current development. 


\section{Appendix A}

For the calculation of $P\left\{X_{1}+X_{2} \leq s\right\}$, we divide the area over which we have to integrate into five parts $A, B, C, D$ and $E$; where $P\{A\}=P\left\{X_{1}+X_{2} \leq s\right.$, $\left.X_{1}>-\frac{s}{2}, X_{2}>\frac{s}{2}\right\}, P\{C\}=P\left\{X_{1} \leq \frac{s}{2}, X_{2} \leq \frac{s}{2}\right\}, P\{D\}=P\left\{X_{1}+X_{2} \leq\right.$ $\left.s, X_{1} \leq-\frac{s}{2}, X_{2}>\frac{s}{2}\right\}$, and where $P\{B\}$ and $P\{E\}$ are the counterparts of $P\{A\}$ and $P\{D\}$ respectively. We start by $P\{C\}$ :

$$
\begin{aligned}
P\{C\} & =P\left\{X_{1} \leq \frac{s}{2}, X_{2} \leq \frac{s}{2}\right\}=P\left\{X_{1} \leq \frac{s}{2}\right\} P\left\{X_{2} \leq \frac{s}{2}\right\} \\
& \approx 1-A_{1}\left(\frac{s}{2}\right)^{-\alpha_{1}}-A_{1} B_{1}\left(\frac{s}{2}\right)^{-\alpha_{1}-\beta_{1}}-A_{2}\left(\frac{s}{2}\right)^{-\alpha_{2}}-A_{2} B_{2}\left(\frac{s}{2}\right)^{-\alpha_{2}-\beta_{2}}
\end{aligned}
$$

as $s \rightarrow \infty$. The terms which are of smaller order, like $s^{-2 \alpha}=s^{-2 \min \left\{\alpha_{1}, \alpha_{2}\right\}}$, can be ignored throughout this proof. The probability $P\{A\}$ takes more effort

$$
\begin{aligned}
P\{A\} & =P\left\{X_{1}+X_{2} \leq s, X_{1}>-\frac{s}{2}, X_{2}>\frac{s}{2}\right\} \\
& =\int_{-s / 2}^{s / 2}\left[F_{2}(s-x)-F_{2}\left(\frac{s}{2}\right)\right] f_{1}(x) d x \\
& =\int_{-s / 2}^{s / 2} F_{2}(s-x) f_{1}(x) d x-\int_{-s / 2}^{s / 2} F_{2}\left(\frac{s}{2}\right) f_{1}(x) d x \\
& =I-I I,
\end{aligned}
$$

where $f_{i}(\cdot)$ and $F_{i}(\cdot)$ denote respectively the density function and distribution function of $X_{i}$. For integral $I$ note that a second order Taylor approximation gives

$$
(s-x)^{-\alpha} \approx s^{-\alpha}+\alpha s^{-\alpha-1} x+\frac{(\alpha+1) \alpha}{2} s^{-\alpha-2} x^{2} .
$$


Hence, for large $s$

$$
\begin{gathered}
I \approx\left[1-A_{2} s^{-\alpha_{2}}-A_{2} B_{2} s^{-\alpha_{2}-\beta_{2}}\right] \int_{-s / 2}^{s / 2} f_{1}(x) d x \\
-\left[\alpha_{2} A_{2} s^{-\alpha_{2}-1}+\left(\alpha_{2}+\beta_{2}\right) A_{2} B_{2} s^{-\alpha_{2}-\beta_{2}-1}\right] \int_{-s / 2}^{s / 2} x f_{1}(x) d x \\
-\frac{\left(\alpha_{2}+1\right) \alpha_{2}}{2} A_{2} s^{-\alpha_{2}-2} \int_{-s / 2}^{s / 2} x^{2} f_{1}(x) d x \\
\approx\left[1-A_{2} s^{-\alpha_{2}}-A_{2} B_{2} s^{-\alpha_{2}-\beta_{2}}\right]\left[1-2 A_{1}\left(\frac{s}{2}\right)^{-\alpha_{1}}-2 A_{1} B_{1}\left(\frac{s}{2}\right)^{-\alpha_{1}-\beta_{1}}\right] \\
{\left[\alpha_{2} A_{2} s^{-\alpha_{2}-1}+\left(\alpha_{2}+\beta_{2}\right) A_{2} B_{2} s^{-\alpha_{2}-\beta_{2}-1}\right]\left[E\left[X_{1}\right]-2 \frac{\alpha_{1}}{\alpha_{1}-1} A_{1}\left(\frac{s}{2}\right)^{-\alpha_{1}+1}\right]} \\
-\frac{\left(\alpha_{2}+1\right) \alpha_{2}}{2} A_{2} s^{-\alpha_{2}-2} E\left[X_{1}^{2}\right] .
\end{gathered}
$$

And for part $I I$

$$
\begin{aligned}
I I & =F_{2}\left(\frac{s}{2}\right) \int_{-s / 2}^{s / 2} f_{1}(x) d x \\
& \approx\left[1-A_{2}\left(\frac{s}{2}\right)^{-\alpha_{2}}-A_{2} B_{2}\left(\frac{s}{2}\right)^{-\alpha_{2}-\beta_{2}}\right]\left[1-2 A_{1}\left(\frac{s}{2}\right)^{-\alpha_{1}}-2 A_{1} B_{1}\left(\frac{s}{2}\right)^{-\alpha_{1}-\beta_{1}}\right]
\end{aligned}
$$

Combine the two parts to obtain $P\{A\}$.

$$
\begin{aligned}
P\{A\} & =I-I I \\
& \approx-A_{2} s^{-\alpha_{2}}-A_{2} B_{2} s^{-\alpha_{2}-\beta_{2}} \\
& +A_{2}\left(\frac{s}{2}\right)^{-\alpha_{2}}+A_{2} B_{2}\left(\frac{s}{2}\right)^{-\alpha_{2}-\beta_{2}}-\alpha_{2} A_{2} s^{-\alpha_{2}-1} E\left[X_{1}\right] \\
& -\frac{\left(\alpha_{2}+1\right) \alpha_{2}}{2} A_{2} s^{-\alpha_{2}-2} E\left[X_{1}^{2}\right] .
\end{aligned}
$$


The probability $P\{D\}$ is

$$
\begin{aligned}
P\{D\} & =P\left\{X_{1}+X_{2} \leq s, X_{1} \leq-\frac{s}{2}, X_{2}>\frac{s}{2}\right\} \\
& =\int_{-\infty}^{-s / 2}\left[F_{2}(s-x)-F_{2}\left(\frac{s}{2}\right)\right] f_{1}(x) d x \\
& =O\left(s^{-2 \alpha}\right)
\end{aligned}
$$

Similar expressions hold for $P\{B\}$ and $P\{E\}$.

\section{Appendix B}

Suppose that the tails of the distributions of $X$ satisfy $P\{X>s\}=A s^{-\alpha}\left[1+B s^{-\beta}+o\left(s^{-\beta}\right)\right]$ as $s \rightarrow \infty$, where $\alpha>0, A>0, \beta>0$ and $B$ is a real number. The asymptotic bias for the Hill estimaor $\widehat{1 / \alpha}$ is

$$
E[\widehat{1 / \alpha}-1 / \alpha]=\frac{-B \beta}{\alpha(\alpha+\beta)} s^{-\beta}+o\left(s^{-\beta}\right)
$$

as $s \rightarrow \infty$ in Goldie and Smith (1987). For the portfolio from Case I in Theorem 1, the aymptotic bias of the Hill estimator is

$$
\operatorname{Bias}(\widehat{1 / \alpha})=-\frac{(1-\omega)^{\alpha_{2}}}{\omega^{\alpha_{1}}} \frac{A_{1}}{A_{2}} \frac{\left(\alpha_{2}-\alpha_{1}\right)}{\alpha_{1} \alpha_{2}} s^{-\left(\alpha_{2}-\alpha_{1}\right)}+o\left(s^{-\left(\alpha_{2}-\alpha_{1}\right)}\right)
$$

where

$$
-\frac{(1-\omega)^{\alpha_{2}}}{\omega^{\alpha_{1}}} \frac{A_{1}}{A_{2}} \frac{\left(\alpha_{2}-\alpha_{1}\right)}{\alpha_{1} \alpha_{2}} s^{-\left(\alpha_{2}-\alpha_{1}\right)}<0
$$

which proves the upward bias in the tail estimator $\hat{\alpha}$. 


\section{References}

[1] Arzac, E.R. and Bawa, V.S., 1977. Portfolio choice and equilibrium in capital markets with safety-first investors. Journal of Financial Economics 4, $277-288$.

[2] Bingham, N.H., Goldie, C.M., and Teugels, J.L., 1987. Regular Variation, Cambridge, Cambridge University Press.

[3] Dacarogna, M.M., Müller, U.A., Pictet, O.V. and de Vries, C.G., 2001. Extremal foreign exchange returns in extremely large data sets, Extremes $4: 2,105-127$.

[4] Danielsson, J., de Haan, L., Peng, L. and de Vries, C.G., 2000. Using a bootstrap method to choose the sample fraction in tail index estimation. Journal of Multivariate Analysis 76, 226-248.

[5] Feller, W., 1971. An Introduction to Probability Theory and Its Applications, Vol. II, Wiley, New York.

[6] Geluk, J. and de Haan, L., 1987. Regular variation, extensions and Tauberian theorems. CWI Tract 40, Amsterdam.

[7] Goldie, C.M., Smith, R.L., 1987. Slow varition with remainder: Theory and application. Quarterly Journal of Mathematics Oxford 2nd series 38, $45-71$.

[8] Gourieroux, C., Laurent, J.P. and Scaillet, O., 2000. Sensitivity analysis of values at risk. Journal of Empirical Finance 7, 225-246. 
[9] de Haan, L., and Stadtmüller, U., 1996. Generalized regular variation of second order. Journal of the Australian Mathematical Society, series A 61, 381-395.

[10] Hartmann, P., Straetmans, S. and de Vries, C.G., 2000. Asset Market Linkages in Crisis Periods. Forthcoming Review of Economics and Statistics.

[11] Jansen, D., Koedijk, K.G. and de Vries, C.G., 2000. Portfolio selection with limited downside risk. Journal of Empirical Finance 7, 247-269.

[12] Jansen, D., 2000. Limited downside risk in portfolio selection among U.S. and Pacific Basin equities. mimeo.

[13] Poon, S., Rockinger, M. and Tawn, J., 2003. Nonparametric Extreme Value Dependence Measures and Finance Applications. Statistic Sinca, forthcoming.

[14] Roy, A.D., 1952. Safety first and the holding of assets. Econometrica 20, 431-449.

[15] Susmel, R., 2001. Extreme observations and diversification in Latin American emerging equity markets. Journal of International Money and Finance 20, 971-986.

[16] Straetmans, S., 1998. Extreme Financial Returns and their Comovements. Ph.D. thesis, Tinbergen Institute Research Series, Erasmus University Rotterdam. 
Table 1: Summary statics and Estimates of tail indices

\begin{tabular}{crrrr}
\hline & \multicolumn{2}{c}{ US bonds and stocks } & \multicolumn{2}{c}{ French stocks } \\
& Corporate bonds & \multicolumn{1}{c}{ Stocks } & Thomson-CSF & \multicolumn{1}{c}{ L'Oreal } \\
\hline Mean & 0.004445 & 0.007943 & 0.0000495 & 0.0005861 \\
s.d. & 0.019782 & 0.055702 & 0.01261 & 0.01129 \\
Skewness & 0.746 & -0.488 & -0.239 & 0.061 \\
Kurtosis & 10.027 & 9.888 & 4.114 & 4.311 \\
No. Obs & 804 & 804 & 546 & 546 \\
& & & & \\
$m$ & 16 & 13 & 21 & 13 \\
$X_{(m)}$ & -0.03843 & -0.13150 & 0.0275 & 0.0285 \\
$\alpha$ & 2.932 & 2.601 & 4.370 & 4.829 \\
$q_{1 / 2 n}$ & -0.125 & -0.460 & -0.063 & -0.056 \\
\hline
\end{tabular}

Note: Table 1 and Table 2 are from Jansen et al. (2000). US bond index and a US stock index (1926.01 - 1992.12), Thomson-CSF and L'Oreal, 546 daily observations. $X_{(n-m)}$ denote the $m$-th lowest observation for US assets, the $m$-th largest absolute observation for French stocks, respectively. $q_{\delta}$ denotes VaR level corresponding to the probability $\delta$.

Table 2: Estimated VaR levels corresponding to the stated probabilities

\begin{tabular}{cccc}
\hline Portfolio of two assets & \multicolumn{2}{c}{ US bonds and stocks } & French stocks \\
\hline Probabilities & 0.0025 & 0.000625 & 0.0018 \\
& $(2 / 804)$ & $(0.5 / 804)$ & $(1 / 546)$ \\
\hline $100 \%$ Asset 2 & -0.2695 & -0.4593 & -0.0487 \\
$90 \%$ Asset 2 & -0.2426 & -0.4134 & -0.0438 \\
$80 \%$ Asset 2 & -0.2157 & -0.3675 & -0.0390 \\
$70 \%$ Asset 2 & -0.1888 & -0.3217 & -0.0344 \\
$60 \%$ Asset 2 & -0.1622 & -0.2763 & $-0.0309^{\mathrm{a}}$ \\
$50 \%$ Asset 2 & -0.1361 & -0.2316 & $-0.0305^{*}$ \\
$40 \%$ Asset 2 & -0.1113 & -0.1887 & -0.0338 \\
$30 \%$ Asset 2 & -0.0896 & -0.1505 & -0.0389 \\
$20 \%$ Asset 2 & -0.0752 & -0.1236 & -0.0443 \\
$10 \%$ Asset 2 & $-0.0721^{*}$ & $-0.1163^{*}$ & -0.0499 \\
$0 \%$ Asset 2 & $-0.0780^{\mathrm{a}}$ & $-0.1251^{\mathrm{a}}$ & -0.0554 \\
\hline
\end{tabular}

Note: The values in parentheses denote the expected number of occurrences. Asset 2 for the US case is US stocks and Asset 2 for the French case is the stock of L'Oreal. * indicates the minimum VaR level among available choices on basis of the second order theory, while ${ }^{\text {a }}$ indicates the portfolio weight with the minimum VaR level from Jansen et al. (2000). 
Table 3: Portfolio selection for monthly US stocks and bonds

\begin{tabular}{|c|c|c|c|}
\hline Portfolio & $q_{\delta}(R)$ & $\begin{array}{c}(R-r) /\left(r-q_{\delta}\right) \\
r=1\end{array}$ & $\begin{array}{c}(R-r) /\left(r-q_{\delta}\right) \\
r=1.00303\end{array}$ \\
\hline \multicolumn{4}{|c|}{ Portfolio selection with $\delta=0.0025$} \\
\hline $100 \%$ Stock & $1-0.2695$ & 0.02947 & 0.01802 \\
\hline $90 \%$ Stock & $1-0.2426$ & 0.03130 & 0.01858 \\
\hline $80 \%$ Stock & $1-0.2157$ & 0.03359 & 0.01927 \\
\hline $70 \%$ Stock & $1-0.1888$ & 0.03650 & 0.02014 \\
\hline $60 \%$ Stock & $1-0.1622$ & 0.04034 & 0.02126 \\
\hline $50 \%$ Stock & $1-0.1361$ & 0.04550 & 0.02274 \\
\hline $40 \%$ Stock & $1-0.1113$ & 0.05252 & $0.02462^{\mathrm{a}}$ \\
\hline $30 \%$ Stock & $1-0.0896$ & 0.06133 & 0.02661 \\
\hline $20 \%$ Stock & $1-0.0752$ & $0.06844^{*}$ & $0.02704^{*}$ \\
\hline $10 \%$ Stock & $1-0.0721$ & $0.06648^{\mathrm{a}}$ & 0.02348 \\
\hline $0 \%$ Stock & $1-0.0780$ & 0.05701 & 0.01747 \\
\hline \multicolumn{4}{|c|}{ Portfolio selection with $\delta=0.000625$} \\
\hline $100 \%$ Stock & $1-0.4593$ & 0.01729 & 0.01063 \\
\hline $90 \%$ Stock & $1-0.4134$ & 0.01838 & 0.01096 \\
\hline $80 \%$ Stock & $1-0.3675$ & 0.01971 & 0.01137 \\
\hline $70 \%$ Stock & $1-0.3217$ & 0.02143 & 0.01190 \\
\hline $60 \%$ Stock & $1-0.2763$ & 0.02369 & 0.01258 \\
\hline $50 \%$ Stock & $1-0.2316$ & 0.02675 & 0.01349 \\
\hline $40 \%$ Stock & $1-0.1887$ & 0.03097 & $0.01468^{\mathrm{a}}$ \\
\hline $30 \%$ Stock & $1-0.1505$ & 0.03653 & 0.01606 \\
\hline $20 \%$ Stock & $1-0.1236$ & $0.04162^{*}$ & $0.01670^{*}$ \\
\hline $10 \%$ Stock & $1-0.1163$ & 0.04125 & 0.01480 \\
\hline $0 \%$ Stock & $1-0.1251$ & $0.03553^{\mathrm{a}}$ & 0.01104 \\
\hline
\end{tabular}

Note: * indicates optimal portfolio among available choices on basis of the second order theory, while ${ }^{\text {a }}$ indicates the optimal choice from Jansen et al. (2000). 
Table 4: Portfolio selection for daily French stocks

\begin{tabular}{rcc}
\hline \multicolumn{1}{c}{ Portfolio } & $q_{\delta}(R)$ & $\begin{array}{c}(R-r) /\left(r-q_{\delta}\right) \\
r=1\end{array}$ \\
\hline 100\% L'Oreal & $1-0.048650$ & $0.01209^{\mathrm{a}}$ \\
$90 \%$ L'Oreal & $1-0.043786$ & 0.01218 \\
80\% L'Oreal & $1-0.038953$ & 0.01226 \\
70\% L'Oreal & $1-0.034358$ & $0.01241^{*}$ \\
$60 \%$ L'Oreal & $1-0.030859$ & 0.01211 \\
$50 \%$ L'Oreal & $1-0.030450$ & 0.01037 \\
40\% L'Oreal & $1-0.033801$ & 0.00778 \\
$30 \%$ L'Oreal & $1-0.038869$ & 0.00542 \\
$20 \%$ L'Oreal & $1-0.044338$ & 0.00352 \\
$10 \%$ L'Oreal & $1-0.049873$ & 0.00210 \\
$0 \%$ L'Oreal & $1-0.055415$ & 0.00088 \\
\hline
\end{tabular}

Note: * indicates optimal portfolio among available choices on basis of the second order theory, while ${ }^{a}$ indicates the optimal choice from Jansen et al. (2000). Portfolio selection is done with $\delta=0.0018$. 
Figure 1. US stock and bond index

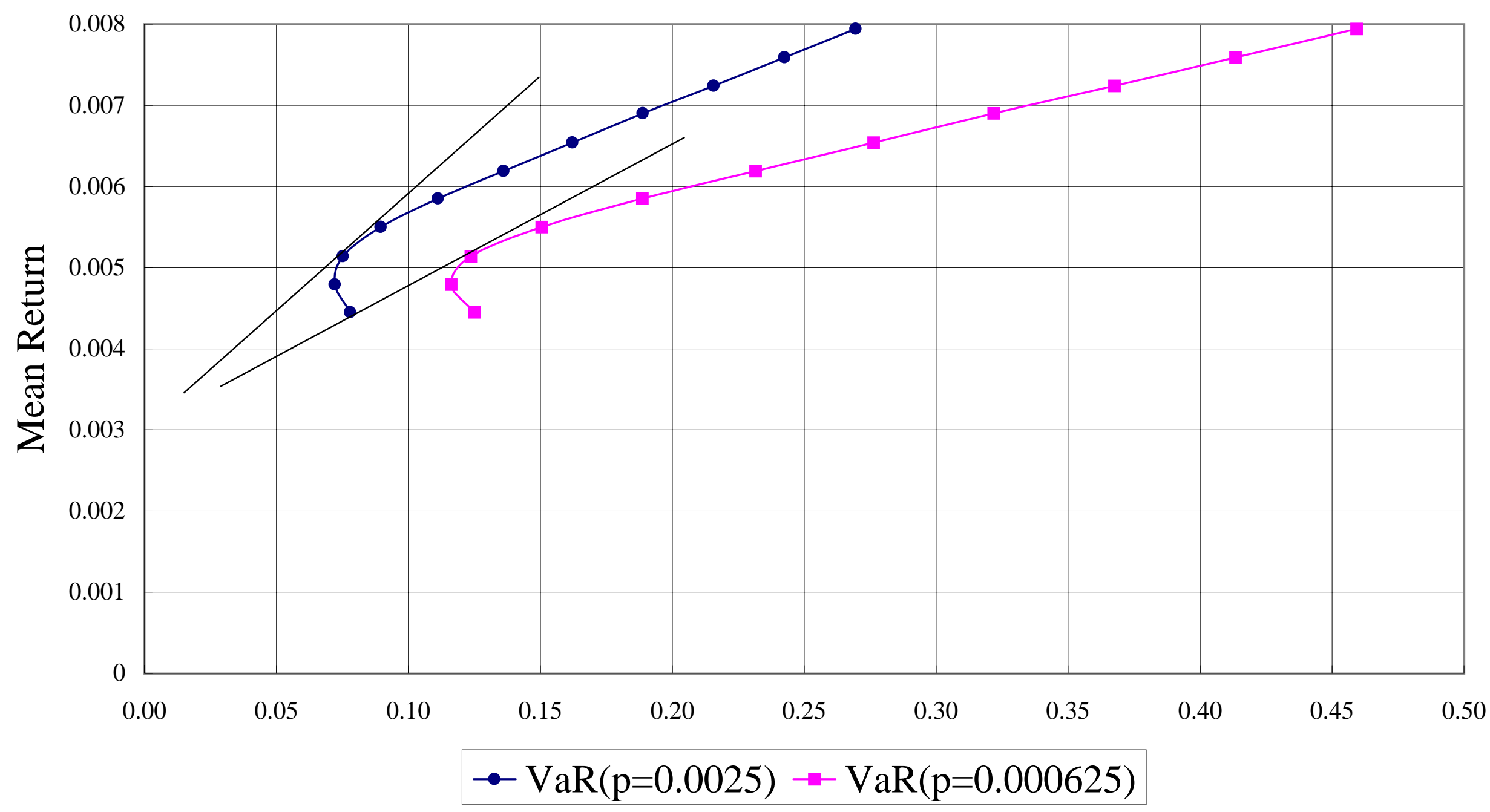


Figure 2. French stocks

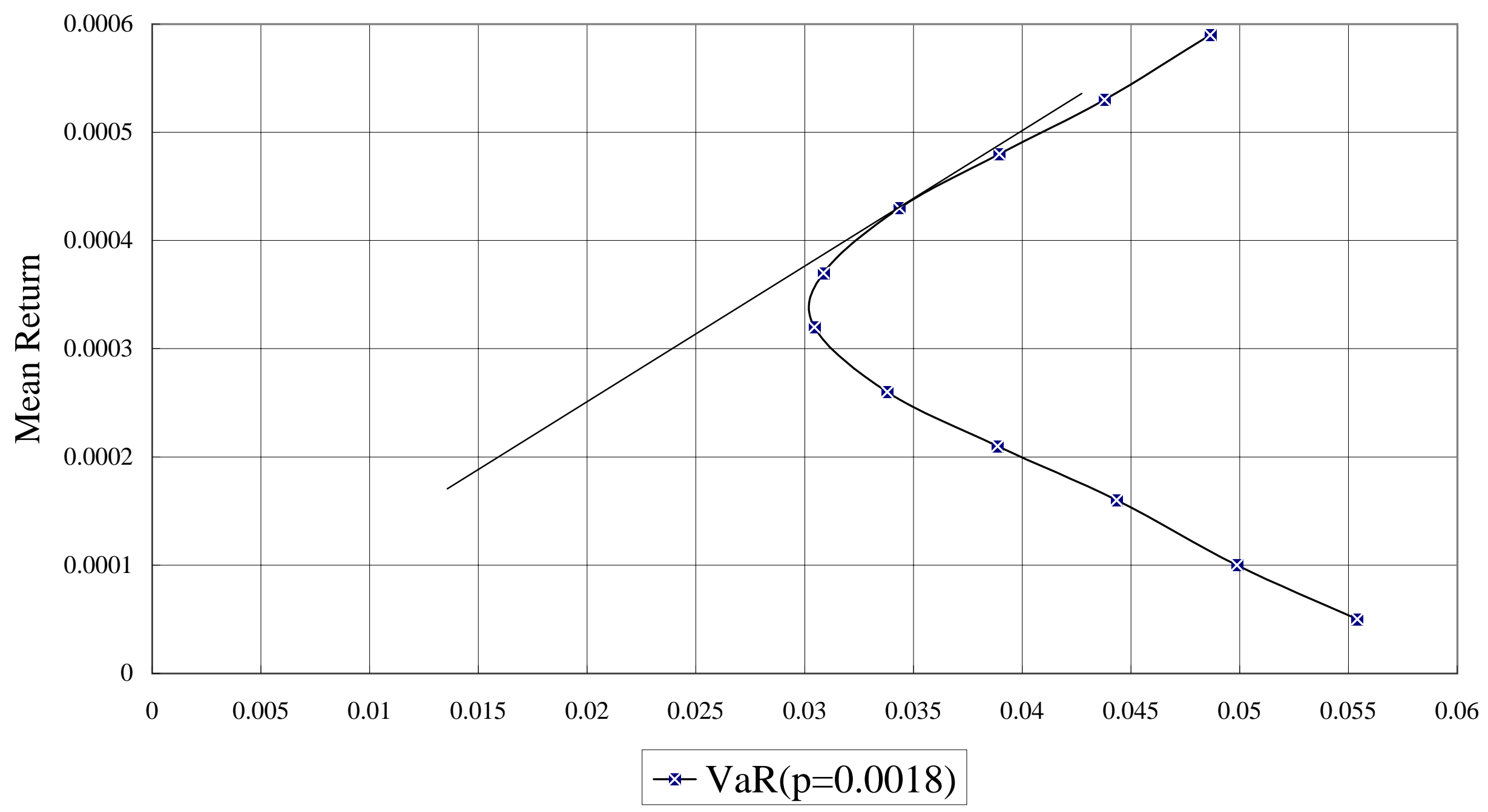

